\title{
Comparison between Windowed FFT and Hilbert-Huang Transform for Analyzing Time Series with Poissonian Fluctuations: A Case Study
}

\author{
Dong $\operatorname{Han}^{1}$ and Shuang-Nan Zhang, 3
}

\begin{abstract}
Hilbert-Huang Transform (HHT) is a novel data analysis technique for nonlinear and non-stationary data. We present a time-frequency analysis of both simulated light curves and an X-ray burst from the X-ray burster 4U 1702-429 with both the HHT and the Windowed Fast Fourier Transform (WFFT) methods. Our results show that the HHT method has failed in all cases for light curves with Poissonian fluctuations which are typical for all photon counting instruments used in astronomy, whereas the WFFT method can sensitively detect the periodic signals in the presence of Poissonian fluctuations; the only drawback of the WFFT method is that it cannot detect sharp frequency variations accurately.
\end{abstract}

Subject headings: - methods: data analysis - stars: oscillations (including pulsations) X-rays: bursts

\section{INTRODUCTION}

In X-ray binaries, matter flows onto the neutron star or black hole through an accretion disk and X-rays are emitted by the accretion flow from inner regions that host the strongest gravitational fields in the Universe. The space time characteristics of the X-ray emitting regions lead to the conclusion that the dynamical time scale for the accretion process of $\mathrm{X}$-ray emitting is millisecond. That means, the millisecond X-ray variability is caused by

\footnotetext{
${ }^{1}$ Department of Engineering Physics \& Center for Astrophysics, Tsinghua University, Beijing 100084; handong@mail.tsinghua.edu.cn

${ }^{2}$ Department of Physics \& Center for Astrophysics, Tsinghua University, Beijing 100084; zhangsn@mail.tsinghua.edu.cn

${ }^{3}$ Key Laboratory of Particle Astrophysics, Institute of High Energy Physics, Chinese Academy of Sciences, Beijing 100049
} 
orbital motion, neutron-star spin, disk- and neutron-star oscillations. So the millisecond Xray variabilities are important for studying strong gravity physics. Kilohertz quasi-periodic oscillations (kHz QPOs), millisecond pulsations and burst oscillations are millisecond X-ray variabilities. Burst oscillations do not occur in every X-ray burst and are different from burst to burst (van der Klis 2000).

Fourier transformation is a traditional method in the time-frequency analysis of linear, stationary and global time series. In astrophysics, Fourier spectral analysis is also widely used in the time-frequency analysis, although time series in astronomy are sometimes non-stationary. Based on the traditional Fourier spectral analysis, Windowed-Fast-Fourier Transform (WFFT) assumes that the time series to be piecewise stationary. In order to result in good time resolution, the window width of WFFT must be narrow; on the other hand, to achieve a high frequency resolution and high detection sensitivity, a wide window width is required. Therefore, in practice the window width must be chosen carefully depending on the goal of the study.

Both $\mathrm{kHz}$ QPOs and burst oscillations were detected in RXTE observations of the atoll source $4 \mathrm{U}$ 1702-429 in 1997. There were six type I X-ray burst, with a rise time of $<1$ s, and a 10-100 s exponential decay. Time-frequency distributions of the light curve from $4 \mathrm{U} 1702-429$ at time resolution $1 / 8192 \mathrm{~s}$ were presented with WFFT with the unit signal $u(t)$ as the window function, and a window width of $2 \mathrm{~s}$. All power spectra were computed by successively sliding the window at a step of $0.125 \mathrm{~s}$ by Markwardt et al. (1999). They discovered burst oscillations at about $330 \mathrm{~Hz}$ in five of the six type I X-ray bursts. In the 1997 July 26 burst a periodic signal was observed whose frequency increased slowly from 328 $\mathrm{Hz}$ to $330 \mathrm{~Hz}$.

The advantages of the Hilbert-Huang Transform (HHT) (see Huang et al. 1998) are that it can be used to analyze nonlinear and non-stationary data without specifying a basis and the data decomposition is made only on physical grounds, compared to Fourier decomposition and wavelet decomposition. Because the light curves obtained with most astronomical instruments involving photon counting devices are characterized by Poissonian fluctuations, it will be very useful if we can analyze such light curves with HHT and obtain detailed physical information such as fast frequency variations. Now, for the light curve of $4 \mathrm{U} 1702-$ 429, the calculated time-frequency distribution and energy-frequency-time distribution using HHT are quite different from those using WFFT. In this paper we shall investigate the large discrepancies from these two methods by making various numerical tests. The structure of the paper is as follows. In section 2, we introduce the concepts and definitions of Fourier frequency and instantaneous frequency in the Hilbert Transform. In section 3, we introduce the HHT. In section 4, we make comprehensive comparisons between the HHT and WFFT. 
Finally we summarize our results and present a discussion in section 5 .

\section{FOURIER FREQUENCY AND INSTANTANEOUS FREQUENCY}

Fourier Transform is very important in time series analysis. The bases of Fourier expansion are trigonometric functions. The resulted frequency from Fourier decomposition is called Fourier frequency.

For an arbitrary time series, $x(t)$, its Hilbert transform $y(t)$ can be expressed as

$$
y(t)=\frac{1}{\pi} \int \frac{x(\tau)}{(t-\tau)} d \tau,
$$

so an analytic signal, $z(t)$, is defined in terms of $x(t)$ and $y(t)$ as

$$
z(t)=x(t)+i y(t)=a(t) e^{i \theta(t)},
$$

where

$$
\begin{gathered}
a(t)=\sqrt{x^{2}(t)+y^{2}(t)} \\
\theta(t)=\arctan \frac{y(t)}{x(t)} .
\end{gathered}
$$

The instantaneous frequency is defined as

$$
f(t)=\frac{1}{2 \pi} \theta^{\prime}(t) .
$$

which is the result of the analytic function by Hilbert transform. It is the first derivative of phase and thus different from the Fourier frequency. The Fourier frequency is constant over the whole time series, while the instantaneous frequency is a function of time. In other words, since all the trigonometric bases of the Fourier transform contribute to Fourier spectrum globally, the Fourier frequency is the global result of the time series within a time span, whereas the instantaneous frequency is a local property of the time series. Thus, only when the time series is composed of pure trigonometric functions, are the Fourier frequencies equal to the instantaneous frequencies, and the instantaneous frequencies of the trigonometric functions constant over the whole time series (Hu 2004).

\section{THE HILBERT HUANG TRANSFORM}

The Hilbert Huang Transform was developed by Huang et al. $(1998,1999)$. In this method any time series, including non-linear and non-stationary series, can be decomposed 
into a finite number of intrinsic mode functions (IMFs) through empirical mode decomposition (EMD) process. An IMF is a function which must satisfy the following two conditions: (1) the difference between the numbers of extrema and zero-crossings is $\leq 1$; and (2) the mean of the upper envelop (linked by local maxima) and the lower envelop (linked by local minima) is zero at every point. With Hilbert transform, the IMFs yield energy-frequencytime distribution, the so-called Hilbert spectrum. HHT has been studied extensively, and has been widely applied in many fields of research. (Nair et al. 2003; Zimmermann \& Seymour 2002; Varadarajan \& Nagarajaiah 2004; Huang et al. 2003)

The EMD process is as follows. According to HHT, once the extrema of a time series $x(t)$ are identified, all the local maxima and minima are connected by two special lines, called the upper and lower envelopes. Their mean is designated as $m_{1}$, and the difference between $x(t)$ and $m_{1}$ is $x(t)-m_{1}=h_{1}$. If $h_{1}$ is not an IMF, $h_{1}$ is treated as the data and undergos the procedure above, giving $h_{1}-m_{11}=h_{11}$. Repeat this sifting procedure $k$ times until $h_{1 k}$ is an IMF, that is $h_{1(k-1)}-m_{1 k}=h_{1 k}$, thus the first IMF component is obtained, i.e. $\mathrm{IMF}_{1}=h_{1 k}$. Then separate $\mathrm{IMF}_{1}$ from the original time series by $x(t)-\mathrm{IMF}_{1}=r_{1}$. Treat $r_{1}$ as the new data and subject it to the same sifting process above. Repeat this procedure on all the subsequent $r_{j}$ s, i.e. $r_{1}-\mathrm{IMF}_{2}=r_{2}, \ldots, r_{n-1}-\mathrm{IMF}_{n}=r_{n}$. So the result is

$$
x(t)=\sum_{j=1}^{n} I M F_{j}(t)+r_{n}(t) .
$$

After Hilbert transform on each IMF, the data can be expressed as

$$
x(t)=\sum_{j=1}^{n} a_{j}(t) \exp \left(i \theta_{j}(t)\right),
$$

then, the instantaneous frequency is

$$
\omega_{j}(t)=\frac{1}{2 \pi} \frac{d \theta_{j}(t)}{d t} .
$$

So the final result is the distribution of the amplitude (of energy) and frequency as the functions of time.

If the time length of $x(t)$ is $T$ and the sampling rate is $\Delta t$, the time resolution is $\Delta t$ and the lowest extractable frequency is $1 / T$, which is also the best frequency resolution. The frequency resolution is up to the Nyquist frequency. 


\section{NUMERICAL COMPARISON BETWEEN WFFT AND HHT}

Different time series, which will be described in the following subsections, are analyzed with HHT-DPS 1.3b, which is a free test-version software from the open NASA internet web site. The digitizing rate of the time series is $1 / 8192 \mathrm{~s}$ and the total time length is $10 \mathrm{~s}$, the same as that of the light curve of the X-ray burst from $4 \mathrm{U} 1702-429$. So the time resolution of the result with HHT is $1 / 8192 \mathrm{~s}$ and the frequency resolution is $0.1 \mathrm{~Hz}$, while the time resolution of WFFT is $0.125 \mathrm{~s}$ and the frequency resolution is $0.5 \mathrm{~Hz}$.

If we set the time and frequency resolutionS of HHT the same as those of WFFT, the time-frequency-energy distribution will be too illegible to reveal anything. The resolution of the computed energy-frequency-time distribution with HHT-DPS $1.3 \mathrm{~b}$ is $400 \times 400$ for the time and frequency axis, i.e. the time span from $0-10 \mathrm{~s}$ is divided into 400 parts, and so is the computed frequency range from 0 to the maximal frequency. The time resolution of the computed time-frequency distribution with HHT-DPS $1.3 \mathrm{~b}$ is $1 / 8192 \mathrm{~s}$, so the illustrated time-frequency distributions with HHT-DPS 1.3b are smoothed with a 205-point average to meet the time resolution of 400 divisions.

\subsection{Comparison between HHT and WFFT with Simple Input Periodic Functions}

We compare between WFFT and HHT using four examples of periodic functions with amplitude set at 3000, approximately the same as the X-ray burst from 4U 1702-429. The input time series and the calculated results with HHT and WFFT are listed in Table 1.

Figure 1 shows the calculated results with HHT for a time series composed of three

Table 1. Testing HHT and WFFT with Simple Input Periodic Functions

\begin{tabular}{|c|c|c|}
\hline Input functions & Results with HHT & Results with WFFT \\
\hline \multicolumn{3}{|l|}{$y=3000 \times(\sin (700 \pi t)+\sin (424 \pi t)$} \\
\hline$+\sin (300 \pi t)+3)$ & Fig. 1 & Left of Fig. 5 \\
\hline Function as above, but with Poissonian sampling. & Fig. 2 & Right of Fig. 5 \\
\hline$y= \begin{cases}3000 \times(\sin (300 \pi t)+1), & \mathrm{t}<5 \mathrm{~s} \\
3000 \times(\sin (700 \pi t)+1), & \mathrm{t}>5 \mathrm{~s}\end{cases}$ & Fig. 3 & Left of Fig. 6 \\
\hline Function as above, but with Poissonian sampling. & Fig. 4 & Right of Fig. 6 \\
\hline
\end{tabular}


sinusoidal functions and a low-level DC-component. Clearly the HHT method failed to detect the three frequencies correctly. For the above times series sampled with Poissonian fluctuations, the HHT method can detect all three sinusoidal components, as shown in Figure 2. However, large spikes in the detected frequencies exist, thus making the instantaneous frequency meaningless. In comparison as shown in Figure 5, the WFFT can detect all three sinusoidal components correctly with or without Poissonian fluctuations as expected.

In Figures 3 and 4, we show the calculated results with HHT for a time series with just one sinusoidal component whose frequency has a sudden jump in the middle of the time series. The HHT method can detect the sinusoidal component correctly; in the presence of Poissonian fluctuations large spikes are again found, however, which make it difficult to judge if the spikes are intrinsic in the time series or simply due to fluctuations. In comparison, as shown in Figure 6, the WFFT method can detect the component correctly without spurious spikes; but the time accuracy of the frequency jump is reduced to about $0.5 \mathrm{~s}$ because of the window applied in doing the FFT.

\subsection{A Case Study of an X-ray Burst from 4U 1702-429 with HHT and WFFT}

We test HHT with the light curve of an X-ray burst from 4U 1702-429 in 1997, which is shown as the solid line in the left panel of Fig. 7. The power spectra (shown as contours) of the original light curve produced with WFFT are also illustrated in the left panel of Figure 7; clearly a periodic component with a varying frequency between $328 \mathrm{~Hz}$ to around $330 \mathrm{~Hz}$ is detected, confirming the results of Markwardt et al. (1999). In comparison, the calculated time-frequency distribution and energy-frequency-time distribution with HHT are shown in Fig. 8. The computed time-frequency distribution with HHT is composed of a series of frequency components with energy generally increasing from low frequency components to high frequency components. The highest frequency about $3000 \mathrm{~Hz}$, due to Poissonian fluctuations, is physically meaningless, but has the largest energy intensity. The presented results with HHT are completely different from those with WFFT from Figure 7 (left panel): (1) There is not an obvious high intensity frequency component at about 330 $\mathrm{Hz}$, as detected with WFFT; (2) The movement of frequencies with time is also different from that with WFFT; and (3) the fluctuations in frequency components make it impossible to reveal any real periodic signals. 


\subsubsection{Test HHT-DPS with synthesized light curves}

To understand why the above calculated results with HHT and WFFT are so different, we take the following steps to find out the conditions at which WFFT or HHT can detect real periodic signals in time series with Poissonian fluctuations.

We eliminate the periodic components in the observed light curve of the X-ray burst, by a smoothening treatment for the light curve through a 25-point average. Then, the smoothed light curve is processed with Poissonian sampling, and the new light curve is analyzed with HHT-DPS 1.3b, in order to understand the behaviors of HHT for Poissonian time series with varying intensities but without periodic signals. To study the minimum strength of periodic signals HHT can detect reliably for the above light curve, a sinusoidal signal is added to the smoothed light curve, and the amplitude and frequency of the added-in sinusoidal signal are both kept constant during the whole time span. Calculations for the synthesized light curves with amplitudes of 0.1 and 0.4 times the mean value of the light curve with a frequency of 400 $\mathrm{Hz}$ are made with HHT. All the results obtained with HHT are compared with those with WFFT. All input synthesized light curves and the calculated results are listed in Table 2.

The calculated results of WFFT agree with our expectations: (1) From the right panel of Figure 7, the original frequency component about $330 \mathrm{~Hz}$ disappeared from the smoothed light curve, and (2) the constant frequency of $400 \mathrm{~Hz}$ can be detected clearly for the two test cases, as shown in Figure 12.

For the results of HHT, comparing Figure 9 with Figure 8, we can see, as expected, that the energy of the frequency components higher than $500 \mathrm{~Hz}$ becomes weaker when the input time series is the smoothed light curve. Figures 10 and 11 show that all frequency components changed when the periodic signal is added in. However, the $400 \mathrm{~Hz}$ frequency component is not recognizable at all in Figure 10, and barely seen in Figure 11, but the large

number and high energy spikes make it impossible to draw any reliable conclusion on the frequency component from the HHT results.

\section{CONCLUSIONS}

We have reached the following conclusions: (1) Poissonian fluctuations cause large spurious spikes in the calculated Hilbert spectra with the HHT method, sO that in all cases the HHT cannot detect periodic signals reliably as long as the time series is essentially Poissonian; and (2) in comparison, the WFFT method is relatively insensitive to Poissonian fluctuations and can detect periodic signals in all cases with high sensitivity, but the time of frequency jump, should it exists, calculated with WFFT is less accurate than that with 
HHT .

Wu \& Huang (2004) established the statistical characteristics of white noise through empirical study on Fourier spectra of the IMFs of the numerical white-noise time series with EMD, and provided a method for finding out which IMF components represent signals and which represent noises. However, as we have shown in this paper it is practically impossible to

eliminate the spurious spikes in the calculated Hilbert spectra with HHT for Poissonian time series, consequently any empirical estimate of the statistical significance on the frequency component does not help in detecting underlying periodic signals.

It is worth noting, however, that it is possible to combine the HHT and WFFT together to extract as much information as possible from observed time series. For example, the WFFT method may be used to identify all frequency components in the time series and study any slow frequency variations. After the WFFT method identified a frequency jump, as shown in Figure 6, the HHT method can be used to identify the exact time of the sudden jump, as shown in Figure 3 and 4.

We thank the anonymous referee for our previous publication (Feng et al. 2004), whose comments on the HHT method motivated the work reported here. We also thank T. P. Li, M. Wu and L. M. Song for many interesting discussions. This study is supported partly by the Special Funds for Major State Basic Research Projects, the Directional Research Grant of the Chinese Academy of Sciences, the National Natural Science Foundation under grant Nos. 10521001 and 10233010, and the Ministry of Education of China.

\section{REFERENCES}

Feng, H., Li, T.P., \& Zhang, S. N. 2004, ApJ, 606 (1): 424

Flandrin, P., Rilling, G., \& Gonçalvè, P. 2003, IEEE Sig. Pro. Letters, 11 (2): 112

Hu G. S. 2004, Tutorial on modern signal analysis, Tsinghua University Press, ISBN: 7-30209507-8/TN212

Huang, N. E., Shen Z., Long, S. R., Wu, M. C., Shih, H. H., Zhangm, Q. N., Yenm N. C., Tung, C. C., \& Liu, H. H. 1998, Proc. R. Soc. Lond, A (1998) 454: 903

Huang, N. E., Shen Z., \& Long, S. R., 1999, Annu. Rev. Fluid Mech, 31: 417

Huang, N. E., Wu, M. C., Long, S. R., Shen, S. S., Qu, W. D., Gloersen, P., \& Fan, K. 2003, Proc. R. Soc. Lond, A (2003) 459: 2317 
Huang, N. E., Wu, M. C., Qu, W. D., Long, S. R., Shen, S. S., \& Zhang, J. 2003, Appl. Stochastic Models Bus. Ind, 19 (3): 245

Markwardt, C. B., Strohmayer, T. E., \& Swank, J. H. 1999, ApJ, 512: L125

Nair, K. K., Kiremidjian, A. S., Lei, Y., Lynch, T. P., \& Law, K. H. 2003, Proceedings of the international conferrence on structral Health Monitoring

van der Klis, M. 2000, Annual Review of Astronomy and Astrophysics, 38: 717

Varadarajan, N., \& Nagarajaiah, S. 2004, Journal of Engineering Mechanics, 130 (4): 451

Wu, Z. H., \& Huang, N. E., 2004, Proc. R. Soc. Lond, A (2004) 460: 1597

Zimmermann, C. A., \& Seymour, R. 2002, Waterway, Port, Coastal and Ocean Engineering, $128(2): 72$ 
Table 2. Comparison between HHT and WFFT with Synthesized Light Curves

\begin{tabular}{lcc}
\hline \hline \multicolumn{1}{c}{ Synthesized light curve } & Results with HHT & Results with WFFT \\
\hline $\begin{array}{l}\text { The 25-point smoothed light curve } \\
\text { with Poissonian sampling. }\end{array}$ & Fig. 9 & Right of Fig. 7 \\
$\begin{array}{l}\text { The smoothed light curve with added-in } \\
\text { signal and Poissonian sampling. }\end{array}$ & Fig. 10 & Left of Fig. 12 \\
$\begin{array}{l}\text { Light curve as above but with } \\
\text { a different amplitude. }\end{array}$ & Fig. 11 & Right of Fig. 12 \\
\hline
\end{tabular}

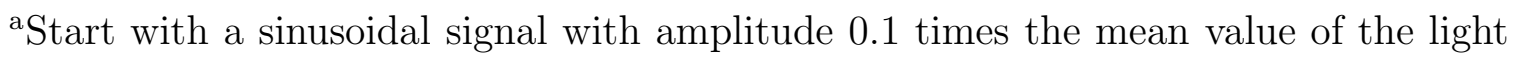
curve, and with frequency set at $400 \mathrm{~Hz}$, add this signal to the smoothed light curve, then sample with Poissonian sampling.

${ }^{\mathrm{b}}$ The same synthesized light curve as above but with a different amplitude of 0.4 times the mean original light curve. 

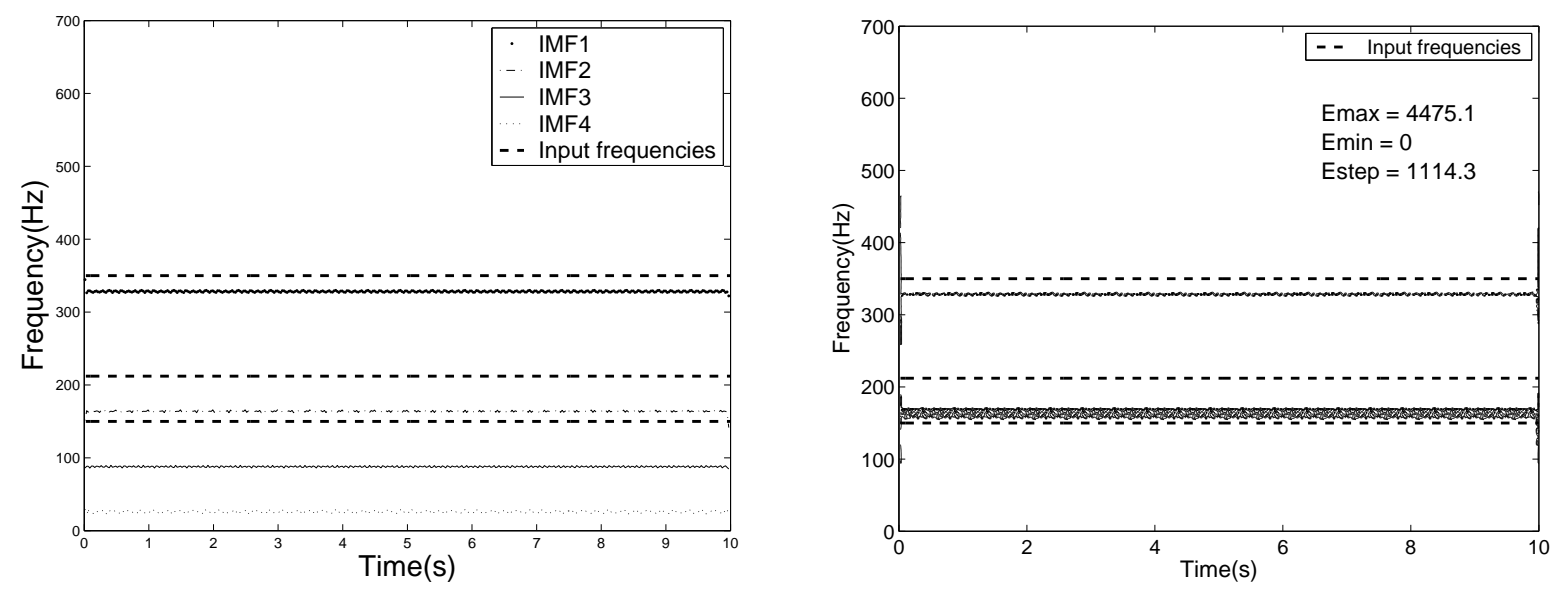

Fig. 1.- Calculated time-frequency distribution (left) and energy-frequency-time distribution (right) with HHT. The input time series is the sinusoidal signal as listed in the first row of Table 1. The dashed lines are for $350 \mathrm{~Hz}, 212 \mathrm{~Hz}$ and $150 \mathrm{~Hz}$, which are the frequencies of the input periodic signals in the time series. For the right panel, the values of the maximum, minimum and step size of the contours are indicated (this is also done in all other contour plots).
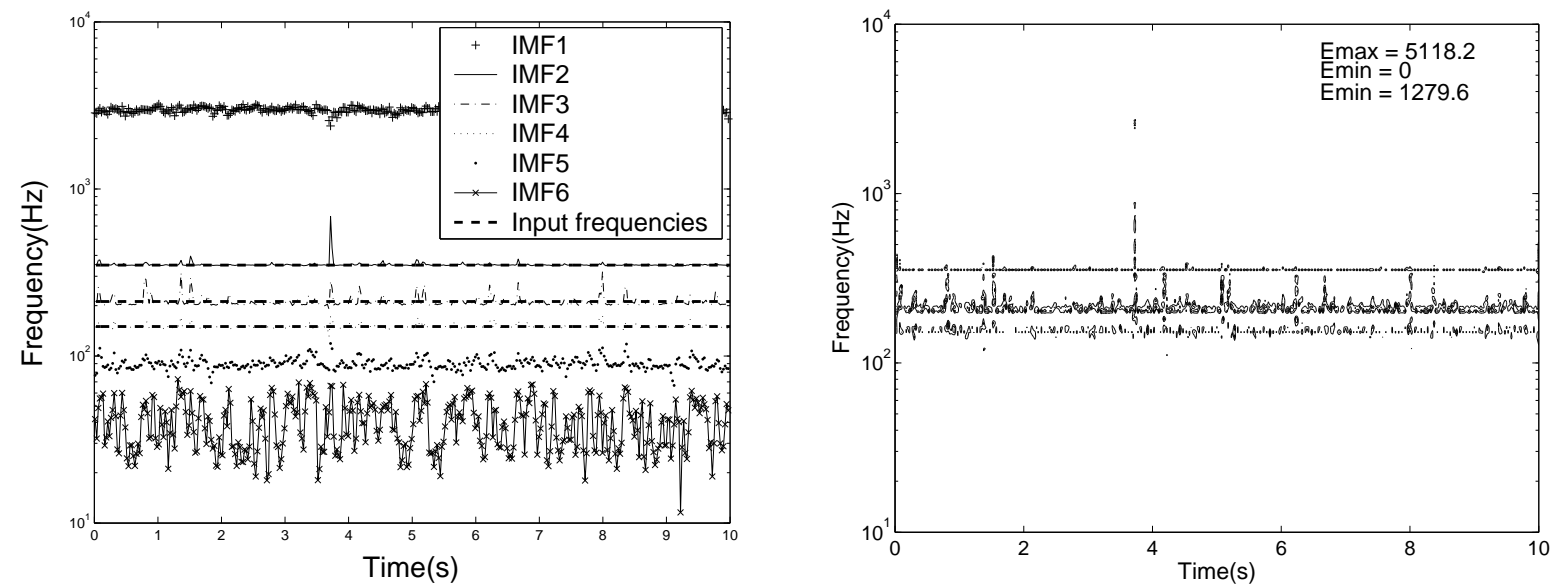

Fig. 2.- Calculated time-frequency distribution (left) and energy-frequency-time distribution (right) with HHT. The input time series is the same as that of Figure 1, but with Poissonian sampling as listed in the second row of Table 1. The dashed lines of the left panel are for $350 \mathrm{~Hz}, 212 \mathrm{~Hz}$ and $150 \mathrm{~Hz}$, which are the frequencies of the input periodic signals in the time series. 

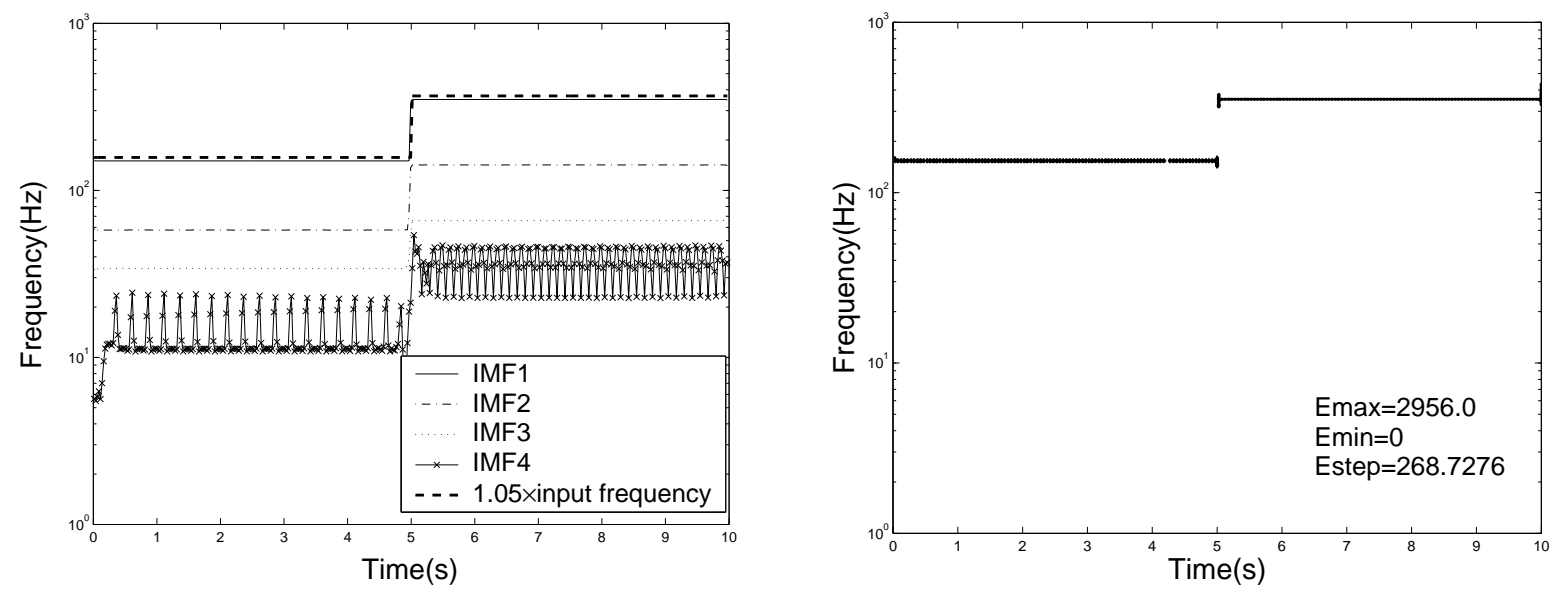

Fig. 3.- Calculated time-frequency distribution (left) and energy-frequency-time distribution (right) with HHT. The input time series is a period signal with just one sinusoidal component whose frequencies have a sudden jump at $5 \mathrm{~s}$ as listed in the third row of Table 1. The dashed line of the left panel is for $1.05 \times$ the frequency of the input periodic signal.
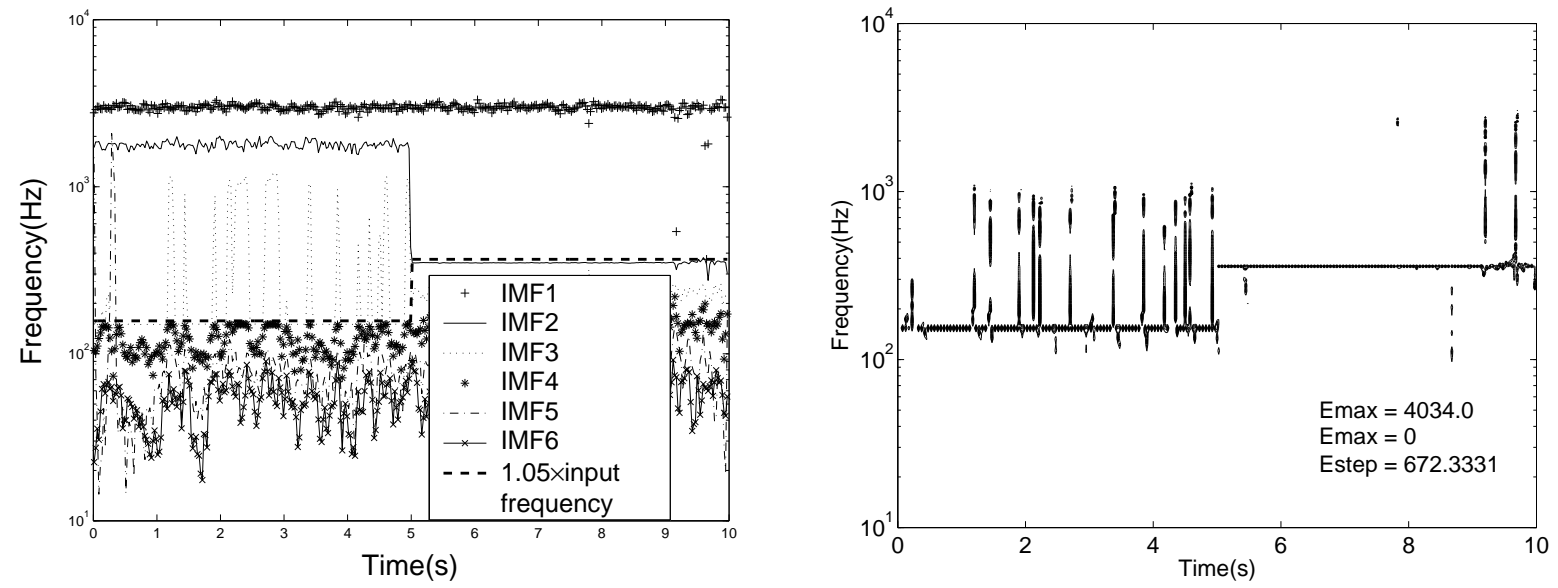

Fig. 4.- Calculated time-frequency distribution (left) and energy-frequency-time distribution (right) with HHT. The input time series is the same as that of Figure 3, but with Poissonian sampling as listed in the forth row of Table 1. The dashed line of the left panel is for $1.05 \times$ the frequency of the input periodic signal. 

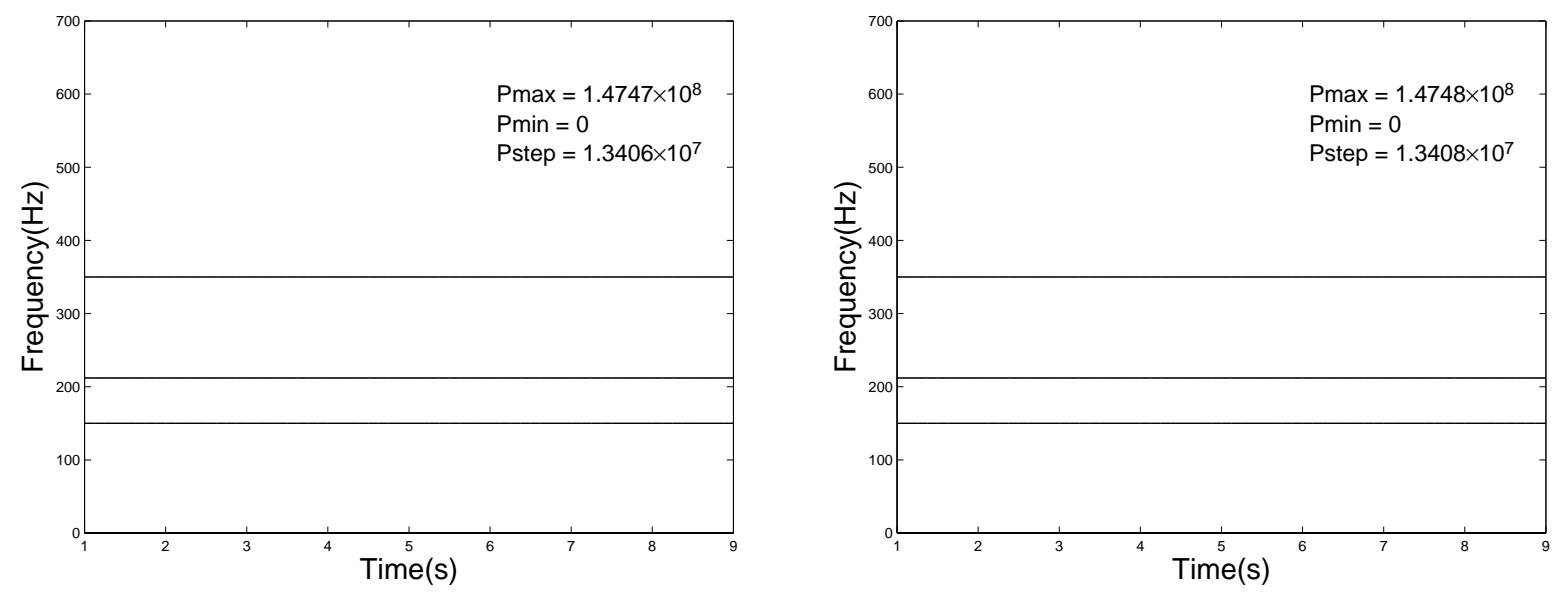

Fig. 5.- Calculated power spectra with WFFT. Left: the result of the input sinusoidal signal as listed in the first row of Table 1. Right: the result of the same time series as that of left panel, but with Poissonian sampling as listed in the second row of Table 1.
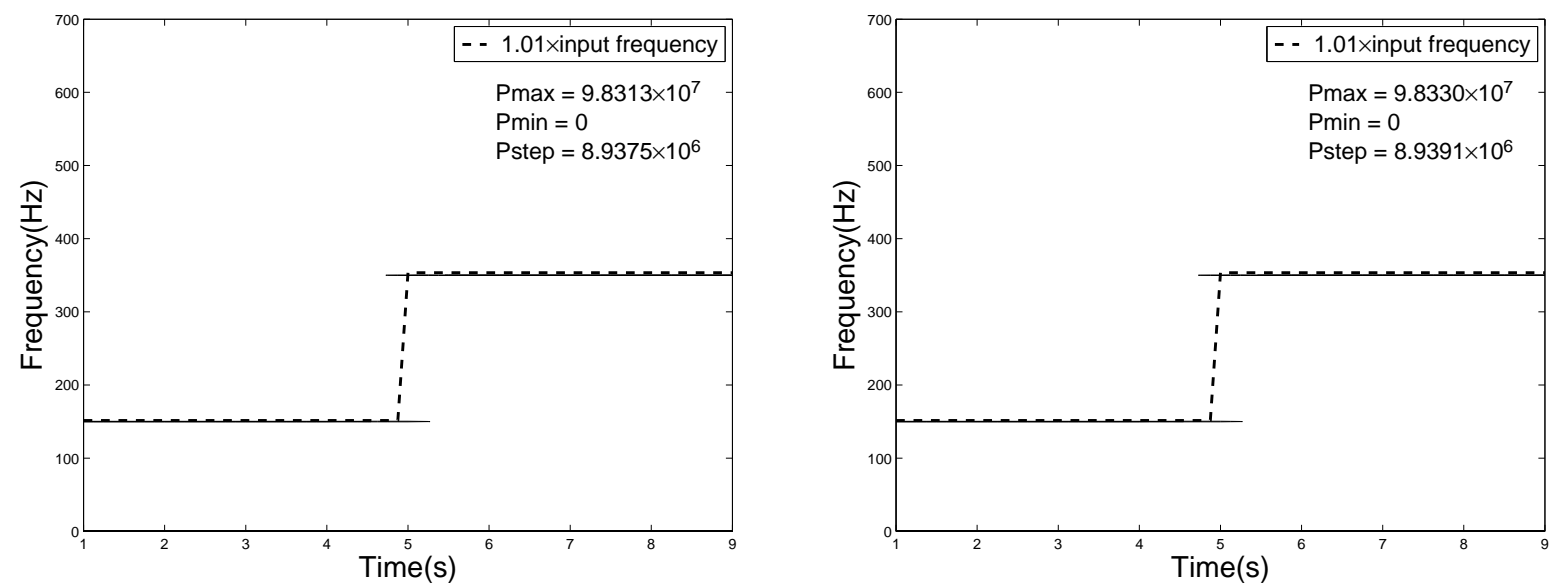

Fig. 6.- Calculated power spectra with WFFT. The dashed line in each panel is for $1.01 \times$ the frequency of the input periodic signal. Left: the result of the input periodic signal whose frequency jumps at $5 \mathrm{~s}$ as listed in the third row of Table 1. Right: the result of the periodic signal with frequency-jump and with Poissonian sampling as listed in the forth row of Table 1 . 

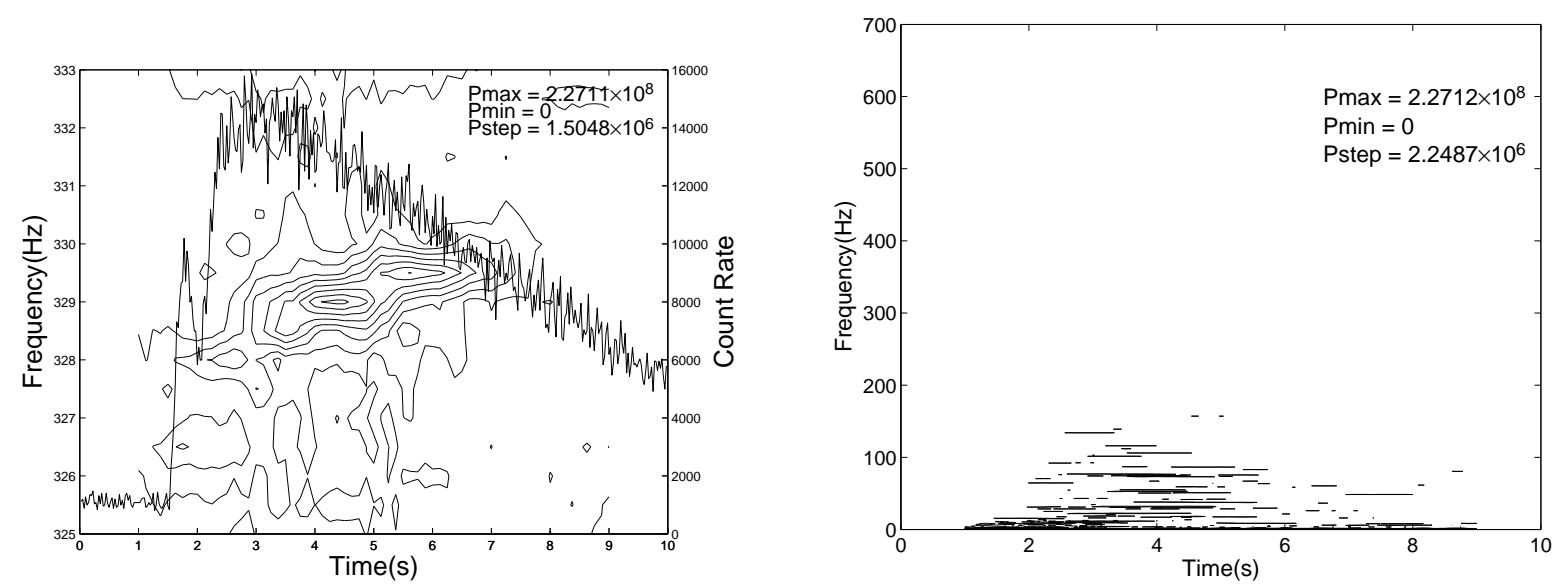

Fig. 7.- Left: light curve (solid line) of the X-ray burst from 4U 1702-429 in 1997, and its power spectra (contours) calculated with WFFT. The component with frequencies about 328 $\mathrm{Hz}$ to $330 \mathrm{~Hz}$ has the most power. Right: power spectra with WFFT for a smoothed light curve. The input time series is the 25-point smoothed light curve with Poissonian sampling as listed in the first row of Table 2. The component with frequencies about $328 \mathrm{~Hz}$ to 330 $\mathrm{Hz}$ shown in the left panel are absent as a result of smoothening.
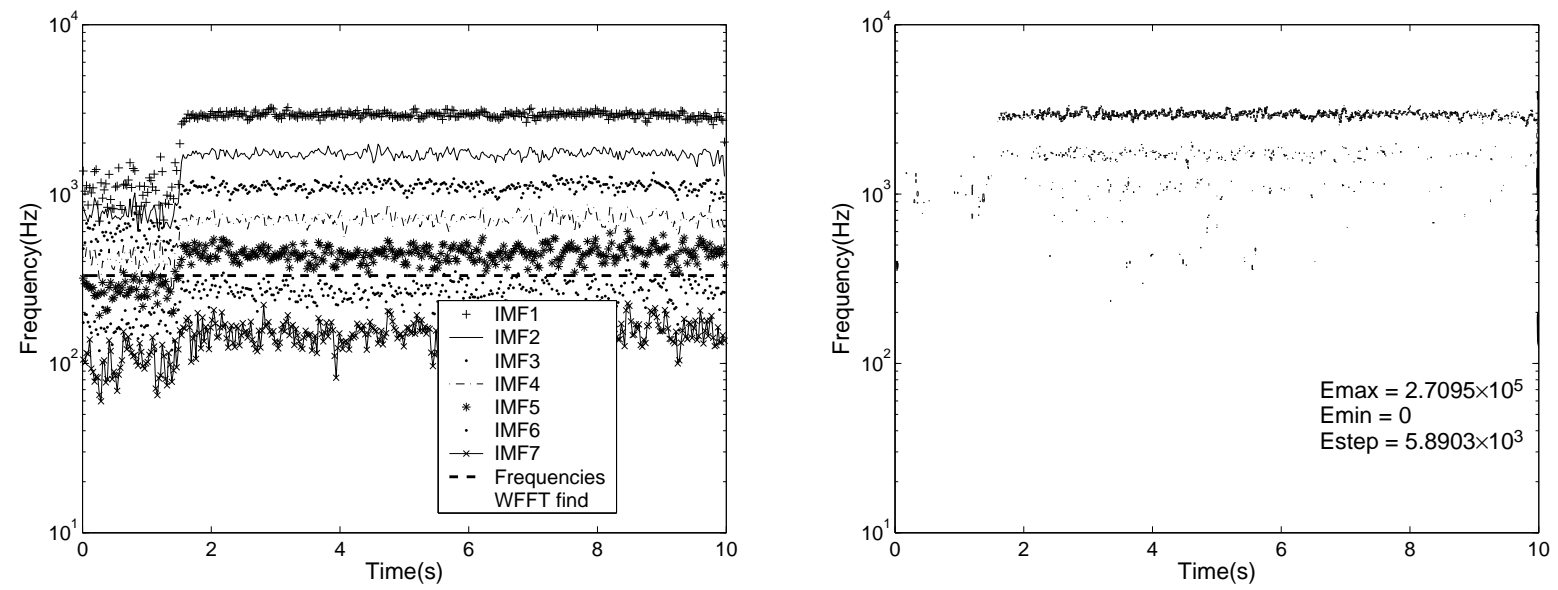

Fig. 8.- Calculated time-frequency distribution (left) and energy-frequency-time distribution (right) with HHT. The dashed line of the left panel is for the $330 \mathrm{~Hz}$ frequency detected with the WFFT method. The input time series is the light curve from 4U 1702-429 in 1997, the same as that of the left panel of Figure 7. The HHT method cannot detect the frequency component around $330 \mathrm{~Hz}$. 

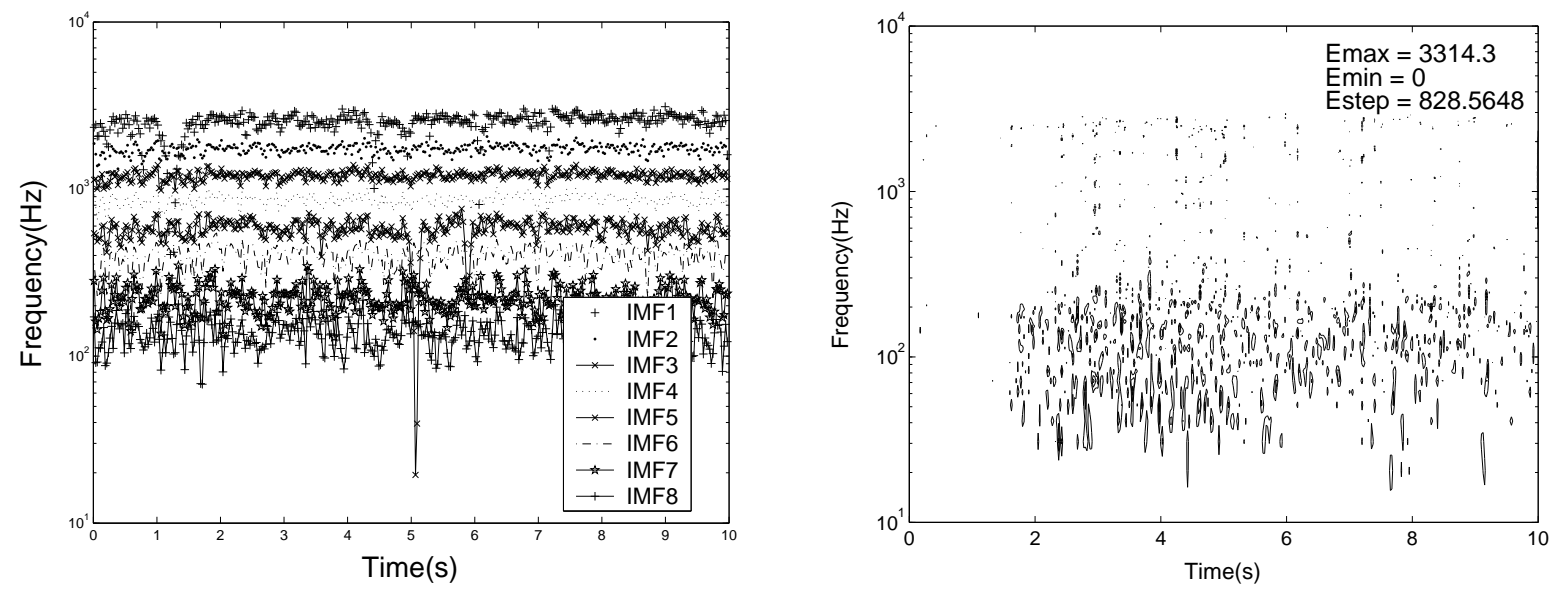

Fig. 9.- Calculated time-frequency distribution (left) and energy-frequency-time distribution (right) with HHT. The input time series is the 25-point smoothed light curve with Poissonian sampling as listed in the first row of Table 2, the same as that of the right panel of Figure 7.
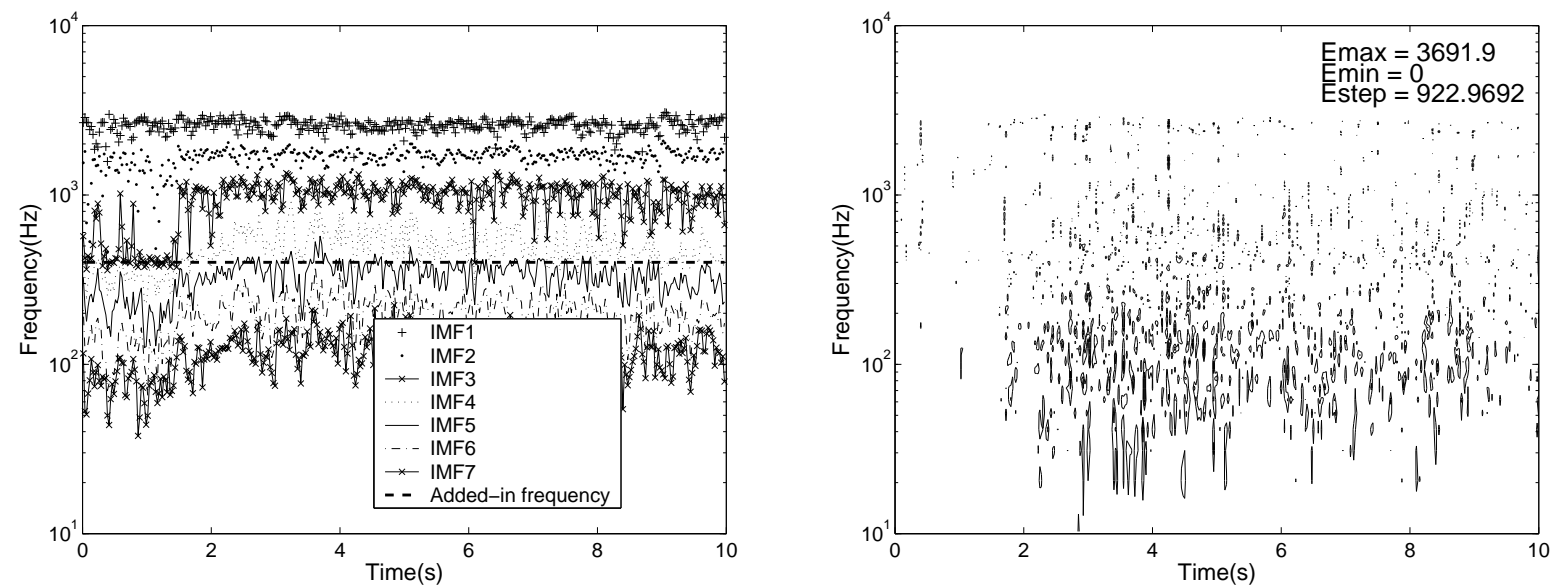

Fig. 10.- Calculated time-frequency distribution (left) and energy-frequency-time distribution (right) with HHT. The input time series is composed of the 25-point smoothed light curve, added with a sinusoidal signal of $400 \mathrm{~Hz}$ and the amplitude of 0.1 times the mean value of the light curve, and then, Poissonian sampling as listed in the second row of Table 2. The dashed line of the left panel is for the $400 \mathrm{~Hz}$ frequency of the added-in sinusoidal signal. 

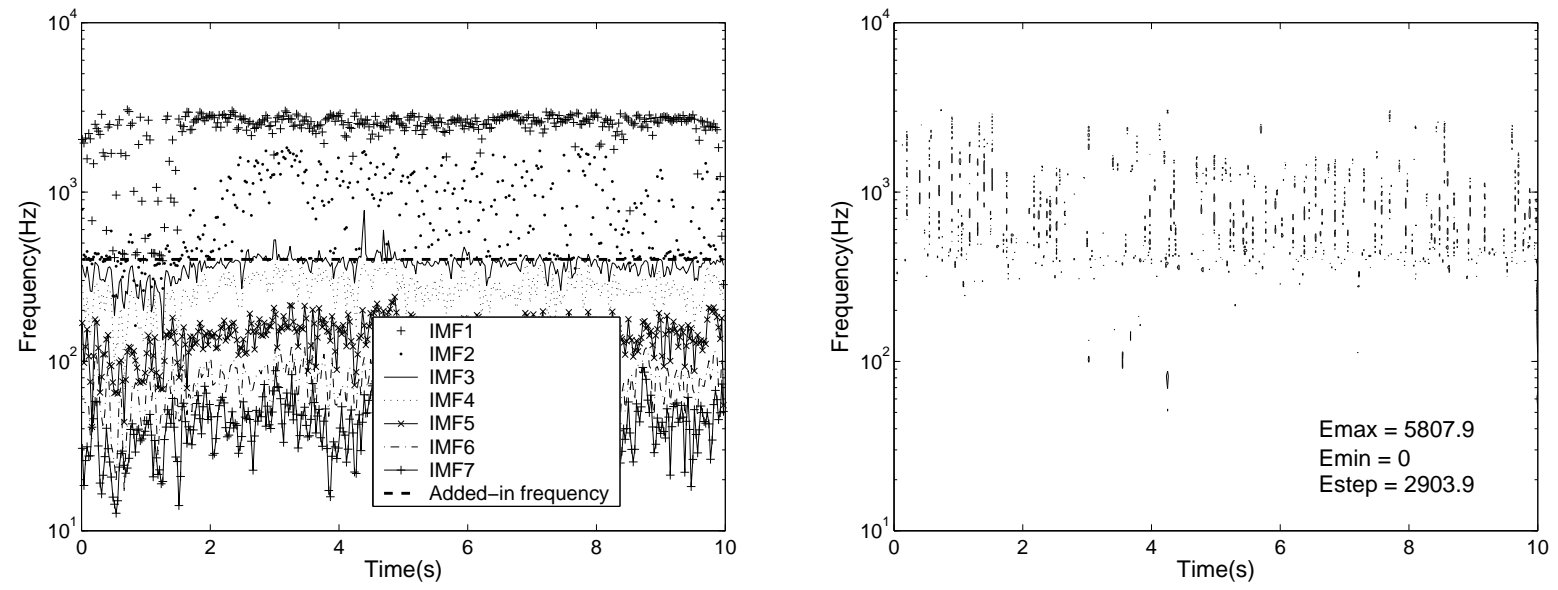

Fig. 11.- Calculated frequency-time distribution (left) and energy-frequency-time distribution (right) with HHT. The input time series is the same as that of Figure 10 but with a different amplitude of 0.4 times the mean value of light curve, as listed in the third row of Table 2. The dashed line of the left panel is for the $400 \mathrm{~Hz}$ frequency of the added-in sinusoidal signal.
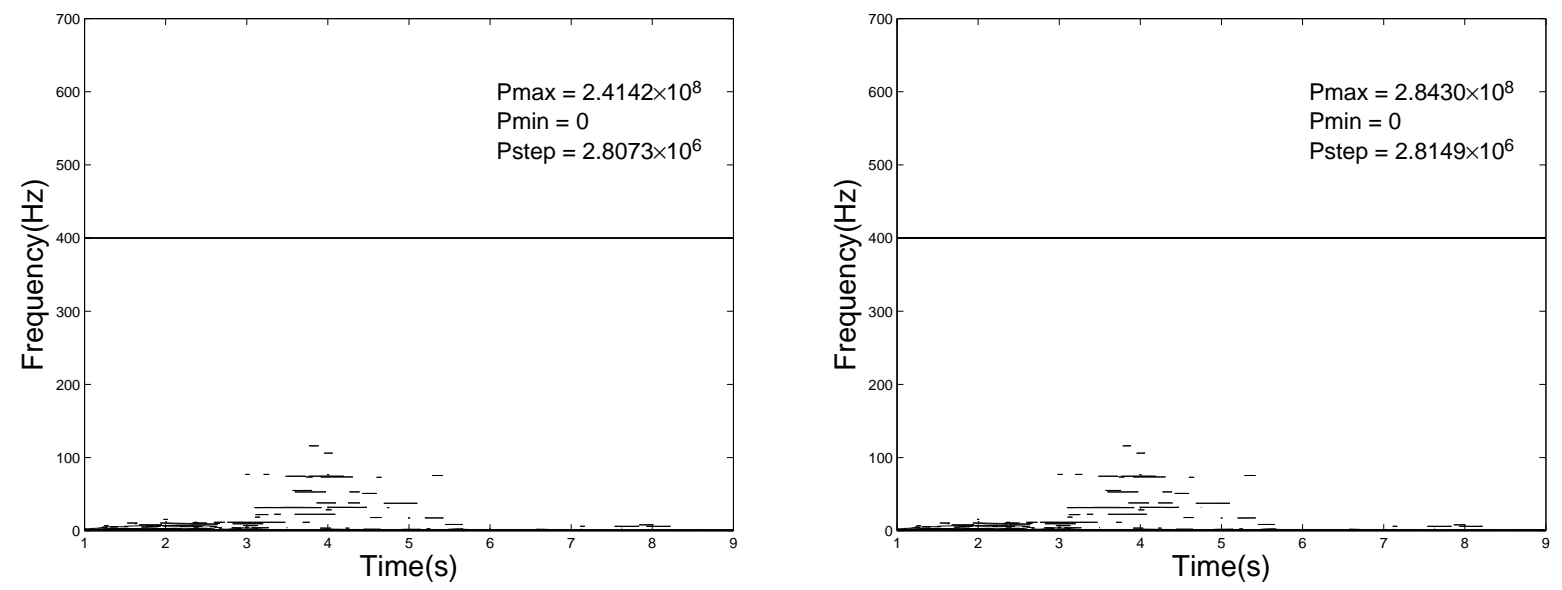

Fig. 12.- Power spectra with WFFT. Left: the input time series is the same as that of Figure 10, as listed in the second row of Table 2. Right: the input time series is the same as that of Figure 11, as listed in the third row of Table 2. 